\title{
Fractional Lévy Motion and Its Application to Network Traffic Modeling
}

\author{
N. Laskin, I. Lambadaris, F. Harmantzis, M. Devetsikiotis
}

N. Laskin is the corresponding author, E-mail address: nlaskin@sce.carleton.ca. N. Laskin and I. Lambadaris are with the Department of Systems and Computer Engineering, Carleton University, Ottawa, K1S 5B6, Canada, Fax: +1 613 520-5727.

F. Harmantzis is with the Department of Electrical and Computer Engineering, University of Toronto, Toronto, ON, M5S 3G4, Canada.

M. Devetsikiotis is with the Department of Electrical and Computer Engineering, NC State University, Raleigh, NC 276957911, USA. 


\begin{abstract}
We introduce a general non-Gaussian, self-similar, stochastic process called the fractional Lévy motion (fLm). We formally expand the family of traditional fractal network traffic models, by including the fLm process. The main findings are the probability density function of the fLm process, several scaling results related to a singleserver infinite buffer queue fed by fLm traffic, e.g., scaling of the queue length, and its distribution, scaling of the queuing delay when independent fLm streams are multiplexed, and an asymptotic lower bound for the probability of overflow (decreases hyperbolically as function of the buffer size).
\end{abstract}

\title{
Keywords
}

Traffic Modeling, Self-Similarity, Heavy-tails, Scaling, Fractal Queuing theory.

\section{INTRODUCTION}

It has been suggested that Internet traffic is far too complicated to be modeled using the techniques developed for the telephone network or computer systems [26]. We argue that although traffic theory currently plays a very minor role in the design of the Internet, it should be increasingly used in the development, design and dimensioning of the future multiservice Internet. Extensive traffic measurement studies from a wide range of data networks and services/applications, have convincingly demonstrated the self-similarity or fractal nature of data traffic [4], [12], [20]. As a consequence, a large number of traffic models have been proposed in order to successfully characterize the real statistical behaviour of the traffic met in networks today. The reason for that is that self-similarity has serious implications for the analysis, design, and control of broadband networks. In contrast, traditional schemes, typically Markovian in nature, which have been (and currently are) extensively used may lead to substantial unterestimation of QoS metrics such as delay and blocking (see [19] for a comprehensive treatment of the problem).

More specifically, measurements and statistical analysis of real traces reveal that traffic exhibits irregularities (burstiness) both in terms of extreme variability of traffic intensities as well as persistent autocorrelation. Such traffic looks extremely irregular (under appropriate aggregation) at different time scales [12] and such extreme behavior is not exhibited by the traditional Poisson traffic which smoothes out when aggregated at coarser time scales. It is said that Internet traffic demonstrates the property of self-similarity. According to Mandelbrot, an irregular, 
self-similar object is called as fractal [14]. Fractal modeling has been used in a number of research areas such as financial mathematics, telecommunications, and chaotic dynamics [1], [17]. Internet traffic and more generally broadband network traffic, is an area where fractal modeling has become popular recently [26].

The first attempt to apply the fractal concept to traffic modeling was to use the of so-called "fractional Gaussian noise" (fGn) instead of traditional Poisson-based models. Compared to standard Gaussian noise, the fractional Gaussian noise model has one extra parameter, the Hurst parameter $H$, which quantifies the strength of the fractal scaling. It is said usually, that the fGn is self-similar, or fractal, with Hurst parameter $H$.

In this paper we formally introduce, develop and elaborate a teletraffic model which takes into account, in addition to the Hurst parameter $H \in[1 / 2,1)$, the Lévy parameter $\alpha \in(1,2]$. It is so-called fractional Lévy motion (fLm), mentioned by Mandelbrot in [15].

Two important subclasses of Lévy motion exist: (i) the well-known ordinary Lévy motion (oLm), an $\alpha$-stable process (distributed in the sense of P. Lévy [13]) with independent increments, which is a generalization of the ordinary Brownian motion (the Wiener process), and (ii) elaborated in this paper, the fractional Lévy motion, a self-similar and stable distributed process, which generalizes the fractional Brownian motion (fBm), has stationary increments and an infinite "span of interdependence".

The (fractional) Lévy random process plays an important role in traffic modeling and more generally in the study of applied stochastic processes, for at least two reasons. The first is that the (fractional) Lévy motion can be considered as a generalization of the (fractional) Brownian motion. The mathematical foundation of the generalization, is obtained using basic properties of stable probability laws. From the limit theorem point of view, stable distributions are generalizations of the widely used Gaussian distributions: Stable distributions are obtained at the limit of (properly normalized) sums of independent identically distributed random variables. An important distinction for the $\alpha$-stable probability distribution is that the power-law (of the complementary cumulative distribution function) decreases as $|x|^{-1-\alpha}$, where $\alpha$ is the Lévy index with $0<\alpha \leq 2$. Hence, the moments of order $\nu \geq \alpha$ diverge. In queuing analysis of telecommunication switches and routers, infinite moments in the input process can engender infinite moments in the queuing process, corresponding to rather long waiting times. 
The second reason for the important role of the fractional Lévy motion is its property of scale invariance or self-similarity. Furthermore, the increments of the process are not only self-similar but also dependent on each other, having at the same time distributions with heavy tails. The fractional Brownian motion is mathematically tractable, and easily applied to modeling of fractal traffic. It is however, a Gaussian process and it has finite variance. The fLm is more general and it may be well suited for modeling of traffic intensities or rates that have large spreadings (in theory infinite variance). Moreover, synthetically generated traces of traffic profiles may be important for stressing/testing actual computer systems/networks. Therefore, we believe that it is important to understand and analyze the behavior of queues fed by fLm traffic streams.

So far, several self-similar stable motions have been proposed for traffic modeling. These processes combine, in a natural way, both scaling behavior and extreme local irregularity. In [9] the authors use the stationary sequence induced by the linear fractional stable motion (LFSM) for heavy-traffic modeling of real Ethernet, VBR video, and WWW traffic. In [6], a comparison study of the queuing performance of the fGn and the noise induced by the LFSM is presented, and in [7] admission control issues are addressed. Consequently, similar models based on other self-similar $\alpha$-stable processes with stationary increments, e.g., $\alpha$-stable Lev́y motion, log-fractional stable motion, have tried to address the problem of self-similarity and heavy-tails at the same time [2], [5], [11]. For more information about these non-Gaussian processes see [23].

The objective of our research in this paper is twofold: a) We provide a formal definition of the fractional Lévy process, show some of its properties, and derive its probability density function following a novel approach, and b) we provide queuing results related to the asymptotic behavior of the tail of the queue-length distribution, the overflow probability, and the queuing delay.

The paper is organized as follows. In Section 2 we present the definition and properties of the fractional Lévy motion (fLm). We start by introducing basic properties of the ordinary Lévy process. Then we define the fLm in terms of a Riemann-Liouville fractional integral and we show the self-similarity of the process and its increments. Further properties of fLm are derived and we conclude with the computation of its probability distribution function and the fractional moments of its increment.

In Section 3 we present a new traffic model based on fLm. Input traffic modeled as fLm, is 
fed to a single server queue with infinite buffer size. Scaling results for the queue size as well as its distribution are then presented. In Section 4, we study the tail distribution of the overflow probability and we calculate an approximate lower bound for it.

In Section 5, we study the scaling dependences of the queuing delay, as the number of multiplexed streams grow in proportion with the service rate of the queue.

Finally in Section 6 we present our conclusions and suggestions for further research.

\section{Fractional LÉvy Motion AND its Properties}

\section{A. Self-Similarity}

There are a number of different definitions of self-similarity. The standard one states that a continuous-time process $Y=\{Y(t), t \geq 0\}$ is self-similar, with self-similarity parameter $H \geq 0(H-s s)$, if it satisfies the condition:

$$
Y(t) \stackrel{d}{=} c^{-H} Y(c t), \forall t \geq 0, \forall c>0
$$

where the equality is in the sense of finite-dimensional distributions. This means that, for any

$d \geq 1$ sequence of time points $t_{1}, \ldots, t_{d}$, and any positive constant $c, c^{-H}\left(Y_{c t_{1}}, Y_{c t_{2}}, \ldots, Y_{c t_{d}}\right)$ has the same distribution as $\left(Y_{t_{1}}, Y_{t_{2}}, \ldots, Y_{t_{d}}\right)$. The Hurst parameter $H$ is the scaling parameter of self-similarity.

The are many different self-similar processes in the literature. We typically consider selfsimilar processes with stationary increments, and call them $H$-sssi processes, since they are of great interest in applications. For details on self-similar processes see [4] and [23]

For example, from the above definition, it is not difficult to check that the Wiener process or (ordinary) Brownian motion (oBm) [4], is a self-similar process with $H=1 / 2$ and since it has stationary increments, it is a $1 / 2$-sssi process.

\section{B. Definition of the fractional Lévy motion}

The counterpart of the Brownian motion for $0<\alpha \leq 2$ is the symmetric $\alpha$-stable Lévy motion ((ordinary) Lévy Motion (oLm)) $L_{\alpha}=\left\{L_{\alpha}(t), t \geq 0\right\}$. oLm is a Markov stochastic process that starts at 0 , has stationary independent increments, and is $H$-sssi with $H=1 / \alpha$, i.e., $L_{\alpha}(c t) \stackrel{d}{=} c^{1 / \alpha} L_{\alpha}(t), t \geq 0$. The probability density function of oLm is 


$$
p_{\alpha}(x, t)=\frac{1}{2 \pi} \int_{-\infty}^{\infty} d k e^{i k x} \exp \left\{-\sigma|k|^{\alpha} t\right\}
$$

where $\sigma>0$ is a scale parameter.

It is known for oLm, that an " $1 / \alpha$ law" can be stated for the fractional structure function $S_{\nu}(\tau, \alpha)=E\left[L_{\alpha}(t+\tau)-L_{\alpha}(t)\right]^{\nu}$ as follows:

For $0<\alpha<2$

1

$$
S_{\nu}(\tau, \alpha)= \begin{cases}\tau^{\nu / \alpha} V(\nu ; \alpha), & \nu<\alpha \leq 2 \\ \infty & \nu \geq \alpha\end{cases}
$$

where $V(\nu ; \alpha)$ is defined as

$$
V(\nu ; \alpha)=\frac{\sigma^{\nu / \alpha}}{2 \pi} \int_{-\infty}^{\infty} d \xi|\xi|^{\nu} \int_{-\infty}^{\infty} d \varsigma \exp \left(i \xi \varsigma-|\varsigma|^{\alpha}\right) .
$$

Following Mandelbrot's generalization of the ordinary Brownian motion to the fractional Brownian motion (fBm) in [15], we define the fractional Lévy motion (fLm) process as the following Riemann-Liouville fractional integral

$$
L_{\alpha, H}(t)=\frac{1}{\Gamma\left(H+\frac{1}{2}\right)} \int_{0}^{t} d L_{\alpha}(\tau)(t-\tau)^{H-1 / 2},
$$

where $L_{\alpha}(t)$ is the ordinary symmetric $\alpha$-stable Lévy Motion (oLm), and $\Gamma(\cdot)$ denotes the gamma function.

Note, that fLm is the generalization of the well known fractional Brownian motion, which can obtained from (5) for $\alpha=2$. So, the role that fLm plays among stable processes is similar to the role that $\mathrm{fBm}$ plays among Gaussian processes.

We also define the increments of the fLm process, as $\Delta L_{\alpha, H}(\tau)=\left\{L_{\alpha, H}(t+\tau)-L_{\alpha, H}(t), \tau \geq\right.$ $0\}$, which is a continuous-time stationary process.

${ }^{1}$ Note, that the $V(\nu ; \alpha)$ can be easily evaluated and as a result we have

$$
V(\nu ; \alpha)=\frac{2 \sigma^{\nu / \alpha}}{\pi \nu} \sin \left(\frac{\pi \nu}{2}\right) \Gamma(1+\nu) \Gamma\left(1-\frac{\nu}{\alpha}\right), \quad \nu<\alpha \leq 2 .
$$

where gamma function $\Gamma(z)$ has a familiar integral representation, $\Gamma(z)=\int_{0}^{\infty} d t t^{z-1} e^{-t}, \operatorname{Re} z>0$. The expression for $V(\nu ; \alpha)$ was obtained at first by West and Seshadri (see Eq.(3.6) in Ref. [25]).

${ }^{2}$ For the definition of the fractional integral see, for example, [?], [22]. 
Let us discuss some important properties of the fLm process and its increments.

Theorem 1: The fLm is a $H$-sssi process with Hurst parameter $H-\frac{1}{2}+\frac{1}{\alpha}$.

Proof: The proof is based on the self-similarity property of oLm.

For $t \geq 0$ and $c>0$,

$$
L_{\alpha, H}(c t)=\frac{1}{\Gamma\left(H+\frac{1}{2}\right)} \int_{0}^{c t} d L_{\alpha}(\tau)(c t-\tau)^{H-1 / 2}
$$

Let $\tau=c s$ and use the fact that $d L_{\alpha}(c s) \stackrel{d}{=} c^{1 / \alpha} d L_{\alpha}(s)$. Then,

$$
\begin{aligned}
L_{\alpha, H}(c t) \stackrel{d}{=} \frac{1}{\Gamma\left(H+\frac{1}{2}\right)} \int_{0}^{c t} c^{1 / \alpha} d L_{\alpha}(s)(c t-c s)^{H-1 / 2} \\
=\frac{c^{H-\frac{1}{2}+\frac{1}{\alpha}}}{\Gamma\left(H+\frac{1}{2}\right)} \int_{0}^{t} d L_{\alpha}(s)(t-s)^{H-1 / 2}=c^{H-\frac{1}{2}+\frac{1}{\alpha}} L_{\alpha, H}(t) .
\end{aligned}
$$

So, according to definition (1), fLm is a $\left(H-\frac{1}{2}+\frac{1}{\alpha}\right)$-sssi process.

Corollary 1: The $\left\{L_{\alpha, H}\left(t_{2}\right)-L_{\alpha, H}\left(t_{1}\right)\right\}$ increment process is self-similar with Hurst parameter $H-\frac{1}{2}+\frac{1}{\alpha}$.

Proof: The proof is based on the self-similarity property of the fLm and the stationarity of its increments. It is easy to show that for $t_{2} \geq t_{1}$ and $c>0$

$$
L_{\alpha, H}\left(c t_{2}\right)-L_{\alpha, H}\left(c t_{1}\right) \stackrel{d}{=} c^{H-\frac{1}{2}+\frac{1}{\alpha}}\left(L_{\alpha, H}\left(t_{2}\right)-L_{\alpha, H}\left(t_{1}\right)\right),
$$

i.e., the increment process is self-similar with the same Hurst parameter $H-\frac{1}{2}+\frac{1}{\alpha}$.

\section{The probability distribution function of the fLm}

In this we derive the probability distribution function (pdf) $p_{\alpha, H}(x, t)$ of the fLm using the pdf of oLm and some results from functional calculus (theory of generalized functions and functionals).

Let us first define the characteristic function $\Phi_{L_{\alpha, H}}(k, t)$ of the fLm as

$$
\Phi_{L_{\alpha, H}}(k, t)=E\left[e^{-i|k| L_{\alpha, H}(t)}\right]=E\left[e^{-i|k| \frac{1}{\Gamma\left(H+\frac{1}{2}\right)} \int_{0}^{t} d L_{\alpha}(\tau)(t-\tau)^{H-1 / 2}}\right]
$$

By taking the inverse Fourier transform of the characteristic function, we can find the $p_{\alpha, H}(x, t)$. 
We now turn our attention to the following lemma, referring to the oLm process.

Lemma 1: For a well behaved function $f(t)$ the following holds

$$
E\left[e^{-i k \int_{0}^{t} d L_{\alpha}(\tau) f(\tau)}\right]=e^{-\sigma|k|^{\alpha} \int_{0}^{t}(f(\tau))^{\alpha} d \tau}
$$

Proof: The proof can be obtained from results from functional calculus and by using the characteristic function of the oLm process, i.e., $E\left[e^{-i k L_{\alpha}(t)}\right]=e^{-\sigma|k|^{\alpha} t}$.

We now derive $p_{\alpha, H}(x, t)$ in the following theorem:

Theorem 2: The probability distribution function (pdf) $p_{\alpha, H}(x, t)$ of the fLm process is given by

$$
p_{\alpha, H}(x, t)=\frac{1}{2 \pi} \int_{-\infty}^{\infty} d k e^{i k x} \exp \left\{-\bar{\sigma}|k|^{\alpha} t^{\alpha\left(H-\frac{1}{2}\right)+1}\right\} .
$$

Proof: Following the above lemma for $f(\tau)=\frac{(t-\tau)^{H-\frac{1}{2}}}{\Gamma\left(H+\frac{1}{2}\right)}$ we get

$$
\Phi_{L_{\alpha}, H}(k, t)=e^{-\sigma \frac{|k| \alpha}{\Gamma^{\alpha}\left(H+\frac{1}{2}\right)} \int_{0}^{t}(t-\tau)^{\alpha\left(H-\frac{1}{2}\right)} d \tau}
$$

or

$$
\Phi_{L_{\alpha, H}}(k, t)=e^{-\bar{\sigma}|k|^{\alpha} t^{\alpha\left(H-\frac{1}{2}\right)+1}},
$$

where

$$
\bar{\sigma}=\frac{\sigma}{\Gamma^{\alpha}\left(H+\frac{1}{2}\right)\left(\alpha\left(H-\frac{1}{2}\right)+1\right)} .
$$

Hence, the $p_{\alpha, H}(x, t)$ is given by equation (7).

By using the Taylor series expansion of the second exponential in (7), we can get

$$
p_{\alpha, H}(x, t)=\delta(x)+\frac{1}{2 \pi} \int_{-\infty}^{\infty} d k e^{i k x} \sum_{n=1}^{\infty} \frac{(-\bar{\sigma})^{n}}{n !}|k|^{\alpha n} t^{\alpha n\left(H-\frac{1}{2}\right)+n}, \quad \alpha<2 .
$$

The integral over $k$ can be evaluated as follows

$$
\begin{gathered}
\frac{1}{2 \pi} \int_{-\infty}^{\infty} d k e^{i k x}|k|^{\alpha n}= \\
\frac{1}{\pi|x|^{\alpha n+1}} \Gamma(\alpha n+1) \cdot \Re e \exp \left(i \frac{\pi(\alpha n+1)}{2}\right)=-\frac{1}{\pi|x|^{\alpha n+1}} \sin \left(\frac{\pi \alpha n}{2}\right) \Gamma(\alpha n+1) .
\end{gathered}
$$

So, the probability density function is equivalent to 


$$
p_{\alpha, H}(x, t)=\delta(x)-\frac{1}{\pi} \sum_{n=1}^{\infty} \frac{(-\bar{\sigma})^{n}}{n !} \frac{t^{\alpha n\left(H-\frac{1}{2}\right)+n}}{|x|^{\alpha n+1}} \sin \left(\frac{\pi \alpha n}{2}\right) \Gamma(\alpha n+1) .
$$

The above series (10) is convenient for the study of the asymptotic behavior of $p_{\alpha, H}(x, t)$, as $|x| \rightarrow \infty$.

The pdf of the fBm is obtained from (7), as a special case for $\alpha=2$ :

$$
p_{2, H}(x, t)=\frac{1}{2 \pi} \int_{-\infty}^{\infty} d k e^{i k x} \exp \left\{-\bar{\sigma} k_{B}^{2} t^{2 H}\right\}=\sqrt{\frac{1}{4 \pi \bar{\sigma}_{B} t^{2 H}}} \exp \left\{-\frac{x^{2}}{4 \bar{\sigma}_{B} t^{2 H}}\right\},
$$

where $\bar{\sigma}_{B}$ is given by (8) for $\alpha=2$, i.e, $\bar{\sigma}_{B}=\sigma / 2 H \Gamma^{2}(H+1 / 2)$.

Corollary 2: A " $\tau^{H-\frac{1}{2}+\frac{1}{\alpha}}$ law" for the fractional structure function of the fLm can be stated as follows:

$$
S_{\nu, H}(\tau, \alpha)=E\left[L_{\alpha, H}(t+\tau)-L_{\alpha, H}(t)\right]^{\nu}= \begin{cases}\tau^{\nu\left(H-\frac{1}{2}+\frac{1}{\alpha}\right)} V_{H}(\nu ; \alpha), & \nu<\alpha \leq 2 \\ \infty & \nu \geq \alpha\end{cases}
$$

where

$$
V_{H}(\nu ; \alpha)=\frac{\bar{\sigma}^{\nu / \alpha}}{2 \pi} \int_{-\infty}^{\infty} d \xi|\xi|^{\nu} \int_{-\infty}^{\infty} d \varsigma \exp \left(i \xi \varsigma-|\varsigma|^{\alpha}\right)
$$

Proof: The proof is be obtained using the pdf of the fLm process.

Proposition 1: If $L_{\alpha, H}(t)$ is $H$-sssi with a continuous fractional structure function of order $\nu$, $1<\nu<\alpha$, then

$$
\frac{1}{2}-\frac{1}{\alpha} \leq H \leq \frac{3}{2}-\frac{1}{\alpha}
$$

Proof: The left part of the inequality can be proved, requiring $\nu$-order moment continuity of $L_{\alpha, H}(t)$ [24]. The right part follows using the " $\tau^{H-\frac{1}{2}+\frac{1}{\alpha}}$ law" and the Minkovski inequality applied to $V_{H}(\nu ; \alpha)$.

\section{QUEUING ANALYSIS WITH FLM INPUT}

In this Section we use the apply the fLm process to define a 4-parameter "fractional Lévy traffic" model, and study the queuing process arising when this traffic is fed to FCFS queue with unlimited buffer space and constant service (leak) rate $r>0$. 


\section{A. The fractional Lévy traffic model}

The "Fractional Lévy traffic" model we consider, is a generalization of the "Fractional Brownian traffic" model that was first introduced by Norros [18]. So, in continuous time, the cumulative traffic (or arrival) process $A(t)$, that is the amount of total load (in bits, say) produced by a source in the time interval $[0, t], t>0$, can be modeled by

$$
A(t)=m t+(\bar{\sigma} m)^{1 / \alpha} L_{\alpha, H}(t)
$$

where $m>0$ is the mean input rate, $\bar{\sigma}$ is the scale factor, and $L_{\alpha, H}(t)$ is the fLm process defined by (5).

The model has four parameters $m, a, \bar{\sigma}$ and $H$ with the following interpretations:

- $m>0$ is the mean constant input rate

- $\alpha \in(1,2]$ measures the "thickness" of the tails of the stable distribution

- $\bar{\sigma}>0$ is the scaling parameter that can be seen as the dispersion around the mean of the traffic - $H \in\left[\frac{1}{2}-\frac{1}{\alpha}, \frac{3}{2}-\frac{1}{\alpha}\right]$ is the Hurst parameter (index of self-similarity)

\section{B. Scaling of the queue length and its distribution}

An important issue is the impact of fractality on queuing. Several network engineering problems, such as buffer dimensioning and traffic control, are related to this question which makes it extremely important.

The first result on queuing analysis of self-similar traffic seems to appear in Norros [18] in which the popular Weibull (lower) bound of the overflow probability has been established using the $\mathrm{fBm}$ input process. In this paper, we elaborate this analysis for the more general case, where the workload is self-similar and stable instead of Gaussian.

Consider a single server queue with constant service rate $r>0$ and infinite buffer space, where the input is a stable self-similar process following (14) $(\rho=m / r$ is the queue utilization, and $r>m$ for stability). The buffer occupancy $Q(t ; r)$ at time $t$ (queue size or queue length), can be written as

$$
Q(t ; r)=\sup _{0 \leq s \leq t}(A(t)-A(s)-r(t-s)),
$$

similarly to the well-known Reich's formula for the virtual waiting time in a queuing system [21], (see also, [3]). 
Hence, $(A(t)-A(s))$ is the amount of work arrived to be processed during the time interval $[s, t]$ and $r(t-s)$ is the amount of work that has been processed in the same time interval.

It is immediately seen that $Q(t ; r)$ is indeed a stationary, fractional stable process. This follows as a consequence of the stationarity, self-similarity and stability of the increments of the fLm process. Scaling dependences of $Q(t ; r)$ can be deduced directly from the properties of fLm. Let us first study the behavior of $Q(t ; r)$ at different time scales.

Theorem 3: The stochastic process $Q(t ; r)$ has the following scaling

$$
Q(c t ; r) \stackrel{d}{=} c^{H-\frac{1}{2}+\frac{1}{\alpha}} Q\left(t ; c^{-H+\frac{3}{2}-\frac{1}{\alpha}} r+\left(1-c^{-H+\frac{3}{2}-\frac{1}{\alpha}}\right) m\right)
$$

for any $c>0$.

Proof: Let

$$
Q(c t ; r)=\sup _{0 \leq s \leq t}(A(c t)-A(c s)-r(c t-c s)) .
$$

Using the fact that the increments of the fLm are self-similar with Hurst parameter $H-\frac{1}{2}+\frac{1}{\alpha}$, we obtain

$$
\begin{gathered}
Q(c t ; r) \stackrel{d}{=} c^{H-\frac{1}{2}+\frac{1}{\alpha}} \sup _{0 \leq s \leq t}\left((\bar{\sigma} m)^{1 / \alpha}\left(L_{\alpha, H}(t)-L_{\alpha, H}(s)\right)+c^{-H+\frac{3}{2}-\frac{1}{\alpha}}(m-r)(t-s)\right)= \\
c^{H-\frac{1}{2}+\frac{1}{\alpha}} Q\left(t ; c^{-H+\frac{3}{2}-\frac{1}{\alpha}} r+\left(1-c^{-H+\frac{3}{2}-\frac{1}{\alpha}}\right) m\right) .
\end{gathered}
$$

In other words, the process $Q(c t ; r)$ is equal in distribution with the $c^{H-\frac{1}{2}+\frac{1}{\alpha}}$ times the original workload process $Q(t ; r)$ with a renormalized service rate $r \rightarrow c^{-H+\frac{3}{2}-\frac{1}{\alpha}} r+\left(1-c^{-H+\frac{3}{2}-\frac{1}{\alpha}}\right) m$. This theorem is the $\alpha$-generalization of the Theorem 3.1 of [18] and includes it in the special case where $\alpha=2$.

Scaling laws with particular significance can be obtained by considering the set of system parameters satisfying the storage threshold exceedance criterion

$$
\epsilon=P(Q(0 ; r)>b)=P\left(\sup _{\tau \geq 0}(A(\tau)-r \tau)>b\right)
$$

Equation (17) can be interpreted as a quality of service (QoS) requirement, defining a storage requirement $b>0$, related to the probability of overflow.

Consider the function

$$
q(b, \varsigma)=P\left(\sup _{\tau \geq 0}\left(L_{\alpha, H}(\tau)-\varsigma \tau\right)>b\right)
$$

So, for $b=1, q(1, \varsigma)=P\left(\sup _{\tau \geq 0}\left(L_{\alpha, H}(\tau)-\varsigma \tau\right)>1\right)$ (strictly decreasing for $\left.\varsigma \geq 0\right)$.

Theorem 4: The function $q(b, \varsigma)$ possess the scaling February 11, 2001 


$$
q(b, \varsigma)=q\left(1, b^{\frac{\frac{3}{2}-\frac{1}{\alpha}-H}{H-\frac{1}{2}+\frac{1}{\alpha}}} \varsigma\right)
$$

Proof: We use only the self-similarity property of the fLm to show this scaling.

$$
\begin{gathered}
q(c b, \varsigma)=P\left(\sup _{\tau \geq 0}\left(\frac{1}{c} L_{\alpha, H}(\tau)-\frac{1}{c} \varsigma \tau\right)>b\right)= \\
P\left(\sup _{\tau \geq 0}\left(L_{\alpha, H}\left(\left(\frac{1}{c}\right)^{1 /\left(H-\frac{1}{2}+\frac{1}{\alpha}\right)} \tau\right)-\frac{1}{c} \varsigma \tau\right)>b\right)= \\
P\left(\sup _{\tau \geq 0}\left(L_{\alpha, H}(\tau)-c^{\frac{\frac{3}{2}-\frac{1}{\alpha}-H}{H-\frac{1}{2}+\frac{1}{\alpha}}} \varsigma \tau\right)>b\right)= \\
q\left(b, c^{\frac{\frac{3}{2}-\frac{1}{\alpha}-H}{H-\frac{1}{2}+\frac{1}{\alpha}}} \varsigma\right) .
\end{gathered}
$$

For $b=1$ we prove the scaling relation.

Taking into account the above scaling result, equation (17) can be rewritten as

$$
\epsilon=q\left(\frac{b}{(\bar{\sigma} m)^{1 / \alpha}}, \frac{r-m}{(\bar{\sigma} m)^{1 / \alpha}}\right)=q\left(1,\left(\frac{b}{(\bar{\sigma} m)^{1 / \alpha}}\right)^{\frac{\frac{3}{2}-\frac{1}{\alpha}-H}{H-\frac{1}{2}+\frac{1}{\alpha}}} \frac{r-m}{(\bar{\sigma} m)^{1 / \alpha}}\right) .
$$

Corollary 3: The QoS requirement in (17) is equivalent to the "bandwidth allocation formula"

$$
r=m+q^{-1}(1, \epsilon) \bar{\sigma}^{1 / \alpha\left(H-\frac{1}{2}+\frac{1}{\alpha}\right)} b^{-\left(\frac{3}{2}-H-\frac{1}{\alpha}\right) /\left(H-\frac{1}{2}+\frac{1}{\alpha}\right)} m^{1 /\left(\alpha\left(H-\frac{1}{2}\right)+1\right)} .
$$

and to the "buffer dimensioning formula"

$$
\frac{1-\rho}{\rho^{1 / \alpha\left(H-\frac{1}{2}+\frac{1}{\alpha}\right)}} b^{\frac{\frac{3}{2}-\frac{1}{\alpha}-H}{H-\frac{1}{2}+\frac{1}{\alpha}}} r^{\frac{H-\frac{1}{2}}{H-\frac{1}{2}+\frac{1}{\alpha}}}=\bar{\sigma}^{1 / \alpha\left(H-\frac{1}{2}+\frac{1}{\alpha}\right)} q^{-1}(1, \epsilon),
$$

Proof: Taking the inverse function of $q(1, \varsigma)$ in (20), the following equation holds

$$
(r-m) b^{\left(\frac{3}{2}-H-\frac{1}{\alpha}\right) /\left(H-\frac{1}{2}+\frac{1}{\alpha}\right)} m^{-1 / \alpha\left(H-\frac{1}{2}+\frac{1}{\alpha}\right)}=\bar{\sigma}^{1 / \alpha\left(H-\frac{1}{2}+\frac{1}{\alpha}\right)} q^{-1}(1, \epsilon),
$$

or

$$
\left.r=m+q^{-1}(1, \epsilon) \bar{\sigma}\right)^{1 / \alpha\left(H-\frac{1}{2}+\frac{1}{\alpha}\right)} b^{-\left(\frac{3}{2}-H-\frac{1}{\alpha}\right) /\left(H-\frac{1}{2}+\frac{1}{\alpha}\right)} m^{1 /\left(\alpha\left(H-\frac{1}{2}\right)+1\right)} .
$$

Substituting $\rho=m / r$ to (21), we obtain the "buffer dimensioning formula".

Let us now apply the above formulae to different type of input traffic: 
(i) The input is modeled as ordinary Brownian motion, i.e., $H=\frac{1}{2}$ and $\alpha=2$. In this case (22) reduces to

$$
b=b(\rho)=\text { const } \cdot \rho \cdot(1-\rho)^{-1} .
$$

(ii) The input is modeled as ordinary Lévy motion, i.e., $H=\frac{1}{2}$ and $0<\alpha \leq 2$. In this case we have

$$
b=b(\rho)=\text { const } \cdot \rho^{\frac{1}{\alpha-1}} \cdot(1-\rho)^{-\frac{1}{\alpha-1}} .
$$

As for ordinary Brownian motion, the service rate $r$ has disappeared from (24).

(iii) In the fBm case $\alpha=2$ and $H>\frac{1}{2}$, the situation is different. ¿From the "buffer dimensioning formula" (22), fixing the service rate $r$ and solving for the buffer requirement $b$ as a function of $\rho$, we obtain

$$
b=b(\rho)=\text { const } \cdot \rho^{1 /(2(1-H))} \cdot(1-\rho)^{-H /(1-H)},
$$

which is the (3.6) result presented in [18].

From the "bandwidth allocation formula" (21), fixing $b$ and solving for $r$, we obtain

$$
r=r(\rho)=\text { const } \cdot \rho^{1 /(2 H-1)} \cdot(1-\rho)^{-H /\left(H-\frac{1}{2}\right)},
$$

i.e., (3.7) of [18].

(iv) The fractional Lévy input, is the more general case. Again, from (22), we express the buffer requirement $b$ as a function of utilization $\rho$

$$
b=b(\rho)=\text { const } \cdot \rho^{1 / \alpha\left(\frac{3}{2}-\frac{1}{\alpha}-H\right)} \cdot(1-\rho)^{-\frac{H-\frac{1}{2}+\frac{1}{\alpha}}{\frac{3}{2}-\frac{1}{\alpha}-H}} .
$$

This is $\alpha$-generalization of the result (3.6) in [18].

In order to have have the $\alpha$-generalization of the result (3.7) in [18], from (21)

$$
r=r(\rho)=\text { const } \cdot \rho^{1 / \alpha\left(H-\frac{1}{2}\right)} \cdot(1-\rho)^{-\frac{\left(H-\frac{1}{2}+\frac{1}{\alpha}\right)}{\left(H-\frac{1}{2}\right)}} .
$$

In the following section we calculate an asymptotic lower bound for the QoS requirement $\epsilon$.

\section{Asymptotic LOWER Bound FOR THE PROBABILITy OF BUFFER OVERFLOW}

In this section we present a technique which leads to the calculation of an asymptotic lower bound of the QoS requirement (17). Our approach is different of the ones used in the literature, i.e., [2], [5], [8], [11], since we use the power series expansion of the probability distribution function of the fLm. 
The following theorem provides a lower bound of the complementary distribution function of the queue length:

Theorem 5: An asymptotic lower bound for the overflow probability is given by

$$
\epsilon=P(Q(0 ; r)>b) \geq \Delta_{\alpha} b^{-\alpha\left(\frac{3}{2}-H-\frac{1}{\alpha}\right)}, \quad b \rightarrow \infty
$$

where

$$
\Delta_{\alpha}=M_{\alpha}(\bar{\sigma} m)\left(\frac{\left(\frac{3}{2}-H\right) \alpha-1}{\alpha}\right)^{\alpha}\left(\frac{\alpha\left(H-\frac{1}{2}\right)+1}{\left[\left(\frac{3}{2}-H\right) \alpha-1\right](r-m)}\right)^{\alpha\left(H-\frac{1}{2}\right)+1},
$$

and

Proof:

$$
M_{\alpha}=\frac{\bar{\sigma}}{\alpha \pi} \Gamma(\alpha+1) \sin \frac{\pi \alpha}{2}
$$

Starting from (17) we have

$$
\epsilon=P(Q(0 ; r)>b) \geq \max _{\tau \geq 0} P\left(L_{\alpha, H}(\tau)-\frac{(r-m)}{(\bar{\sigma} m)^{1 / \alpha}} \tau>\frac{b}{(\bar{\sigma} m)^{1 / \alpha}}\right) .
$$

Using the probability distribution function of the fLm $p_{\alpha, H}(x, t)$, one can express the probability $P\left(L_{\alpha, H}(\tau)>B\right)$ as

$$
P\left(L_{\alpha, H}(\tau)>B\right)=\int_{B}^{\infty} d x p_{\alpha, H}(x, t) .
$$

In order to calculate the above probability as $B \rightarrow \infty$, we use only the first term of the series (10), i.e.,

$$
P\left(L_{\alpha, H}(\tau)>B\right) \simeq M_{\alpha} \frac{\tau^{\alpha\left(H-\frac{1}{2}\right)+1}}{B^{\alpha}}, \quad B \rightarrow \infty,
$$

where $M_{\alpha}$ is given by (31).

Hence, for $B=\frac{(r-m) \tau+b}{(\bar{\sigma} m)^{1 / \alpha}}$ we get

$$
P(Q(0 ; r)>b) \simeq \max _{\tau \geq 0}\left\{M_{\alpha}(\bar{\sigma} m) \frac{\tau^{\alpha\left(H-\frac{1}{2}\right)+1}}{((r-m) \tau+b)^{\alpha}}\right\}, \quad b \rightarrow \infty .
$$

By differentiating the expression in the right side of the above equation, we find that the maximum is obtained for $\tau=\tau_{0}$, where

$$
\tau_{0}=\frac{\alpha\left(H-\frac{1}{2}\right)+1}{\left[\left(\frac{3}{2}-H\right) \alpha-1\right](r-m)} b
$$


By substituting $\tau_{0}$ to find the maximum, we obtain the asymptotic given by (29), and the proof is complete.

In order to find the required capacity $r$ to satisfy the QoS criterion approximately, we simply solve (29) for $r$, i.e.,

$$
r=m+\left(M_{\alpha} / \epsilon\right)^{1 /\left(\alpha\left(H-\frac{1}{2}\right)+1\right)} \bar{\sigma}^{1 /\left(\alpha\left(H-\frac{1}{2}\right)+1\right)} m^{1 /\left(\alpha\left(H-\frac{1}{2}\right)+1\right)} b^{\frac{H-\frac{3}{2}+\frac{1}{\alpha}}{2-\frac{1}{2}+\frac{1}{\alpha}}} .
$$

Comparing the above approximate requirement with the exact one given by the "bandwidth allocation formula" (21), we see that they differ only by the factor $\left(M_{\alpha} / \epsilon\right)^{1 /\left(\alpha\left(H-\frac{1}{2}\right)+1\right)}$.

The rate of decay in (29) is at most an algebraic function of the buffer size, and is in accordance of the results reported in [5], and [8].

The above result encompasses previous results in the literature, related to traditional traffic models. More specifically,

- For the Brownian case, i.e., $H=1 / 2$ and $\alpha=2$, the expression (29) reduces to the wellknown asymptotic provided by the exponential distribution.

- [11] analyzes a queue with oLm input.

- [18] provides an asymptotic bound for the fBm case.

\section{SCALINGS FOR QUEUING DELAY IN THE FLM CASE}

In this section we explore the queuing delay in a queue fed by fLm traffic, under various scaling regimes. This study was motivated by the consequences of rapidly growing communications capacity for the evolution of the Internet. For example, Kelly [10] argues that queuing delays become small in comparison with propagation delays, giving new insights towards a self-managed Internet.

We assume that traffic and capacity grow proportional in a queuing system, and we follow the same notion as [10], to show the reader the generalized delay results for the fLm case.

Let $a$ be the the volume scaling parameter, $b$ and $c$ the speed and multiplexing parameters: the original workload at the interval $[s, t]$, i.e., $A(t)-A(s)$, has been increased in volume by $a$, speeded up by $b$, and $c$ i.i.d. fLm streams $A_{i}(t), i=1,2, \cdots, c$ have been multiplexed. Therefore, the workload obtained after the scaling in the three regimes is

$$
\sum_{i=1}^{c} a\left(A_{i}(b t)-A_{i}(b s)\right) .
$$

The buffer occupancy at time $t$ of the new system becomes 


$$
Q(t ; a, b, c ; r)=\sup _{0 \leq s \leq t}\left(\sum_{i=1}^{c} a(\bar{\sigma} m)^{1 / \alpha}\left(L_{\alpha, H}^{(i)}(b t)-L_{\alpha, H}^{(i)}(b s)\right)-a b c(r-m)(t-s)\right),
$$

when the new workload is applied to (15).

Since $Q(t ; a, b, c ; r)$ describes the buffer occupancy, the queuing delay under the FIFO discipline is (on average)

$$
\Delta(a, b, c)=Q(a, b, c) / a b c r
$$

As Kelly [10] points out, the impact of the parameters $a$ and $b$ parameters are straight-forward:

$$
\Delta(a, b, c) \stackrel{d}{=} \Delta(1, b, c), \quad \Delta(a, b, c) \stackrel{d}{=} b^{-1} \Delta(a, 1, c) .
$$

In the following theorem we show how the multiplexing parameter impacts the queuing delay (obviously, $\Delta(a, b, c) \leq \Delta(a, b, 1)$ ).

Theorem 6: The queuing delay of the multiplexed fLm streams obeys the following scaling

$$
\Delta(a, b, c) \stackrel{d}{=} c^{-(\alpha-1) /\left(\alpha\left(\frac{3}{2}-H\right)-1\right)} \Delta(a, b, 1) .
$$

Proof: By multiplying $\Delta(a, b, 1)$ by $c^{-(\alpha-1) / \alpha\left(\frac{3}{2}-H\right)-1}$ and using the definitions (33) and (34) we get

$$
\begin{gathered}
c^{-(\alpha-1) / \alpha\left(\frac{3}{2}-H\right)-1} \Delta(a, b, 1) \stackrel{d}{=} \\
\sup _{0 \leq s \leq t}\left(c^{-(\alpha-1) / \alpha\left(\frac{3}{2}-H\right)-1} \frac{(\bar{\sigma} m)^{1 / \alpha}\left(L_{\alpha, H}(b t)-L_{\alpha, H}(b s)\right)}{b r}-c^{-(\alpha-1) / \alpha\left(\frac{3}{2}-H\right)-1} \frac{(r-m)}{r}(t-s)\right) .
\end{gathered}
$$

Further, changing the time variables $t$ and $s$ as

$$
c^{-(\alpha-1) / \alpha\left(\frac{3}{2}-H\right)-1} \cdot(t, s) \rightarrow t^{\prime}, s^{\prime}
$$

yields

$$
\begin{gathered}
c^{-(\alpha-1) / \alpha\left(\frac{3}{2}-H\right)-1} \Delta(a, b, 1) \stackrel{d}{=} \\
\sup _{0 \leq s^{\prime} \leq t^{\prime}}\left(\frac{c^{-(\alpha-1) / \alpha}(\bar{\sigma} m)^{1 / \alpha}\left(L_{\alpha, H}\left(b t^{\prime}\right)-L_{\alpha, H}\left(b s^{\prime}\right)\right)}{b r}-\frac{(r-m)}{r}\left(t^{\prime}-s^{\prime}\right)\right),
\end{gathered}
$$

where the self-similar property of fLm was taken into account.

From the additive property of the stable processes, e.g., [23], we have that the superposition of $c$ fLm streams with the same scale parameter $\bar{\sigma}$ leads to renormalization of the scale by factor $c^{1 / \alpha}$, i.e., $\bar{\sigma} \rightarrow c^{1 / \alpha} \bar{\sigma}$. Hence, we obtain 


$$
\begin{gathered}
c^{-(\alpha-1) / \alpha\left(\frac{3}{2}-H\right)-1} \Delta(a, b, 1) \stackrel{d}{=} \\
\sup _{0 \leq s \leq t}\left(\frac{\sum_{i=1}^{c} a(\bar{\sigma} m)^{1 / \alpha}\left(L_{\alpha, H}^{(i)}(b t)-L_{\alpha, H}^{(i)}(b s)\right)}{a b c}-\frac{(r-m)}{r}(t-s)\right) .
\end{gathered}
$$

It is easy to see that the right side of the last equation equals $Q(t ; a, b, c ;) / a b c$, i.e., $\Delta(a, b, c)$.

\section{CONCLUSIONS}

In this paper, we extend Mandelbrot's work [15], dropping the assumption of finite variance and falling into the stable $H$-sssi processes as the next larger class. We introduce fractional Lev́y motion (fLm), which attains integral representation similar to fractional Brownian motion (fBm) [15], keeping the same exponents $H-\frac{1}{2}$ for the integration kernel, and using the ordinary symmetric Lev́y motion as an integrating process rather than ordinary Brownian motion. While there are several other fractional stable motions in the literature [23], this is the first time that fLm and its probability density function (or characteristic function) are introduced.

Based on fLm, we develop a parsimonious traffic model, suitable for traffic modeling in modern broadband networks. Since empirical data collected for a variety of communication networks and applications exhibit self-similarity and heavy-tailed dependences, it is reasonable to apply the "Fractional Lev́y traffic" model which captures these characteristics. Statistical analysis of TCP traces and preliminary results justify our argument, and fLm seems to be quite promising in describing the observed properties and behavior of today's teletraffic.

We further elaborate all the well-known fractal queuing results obtained for Gaussian processes. Both the scaling expressions and the lower asymptotic bound for the overflow probability that are presented here encompass all results in the literature related to $\mathrm{fBm}$ and oLm.

\section{REFERENCES}

[1] R.J. Adler, R.E. Feldman and M.S. Taqqu. A Practical Guide to Heavy Tails: Statistical Techniques and Applications. Birkhauser, Boston, 1998.

[2] S. Bates. Traffic Characterization and Modeling for Call Admission Control Schemes on ATM networks. PhD dissertation, University of Edinburgh, UK, 1997.

[3] V.E. Benes. General Stochastic Processes in the Theory of Queues. Addison-Wesley, 1963. 
[4] J. Beran. Statistics for Long-Memory Processes. Chapman \& Hall, New York, 1994.

[5] J.R. Gallardo, D. Makrakis, and L. Orozco-Bardosa. Use of Alpha-Stable Self-Similar Stochastic Processes for Modeling Traffic in Broadband Networks. Performance Evaluation, Vol. 40, pp. 71-98, 2000.

[6] R.G. Garroppo, S. Giordano, S. Porcarelli and G. Procissi. Testing $\alpha$-Stable Processes in Modelling Broadband Teletraffic. In Proc. ICC'O0, pp. 1615-1619, New Orleans, LA, 2000.

[7] F.C. Harmantzis, D. Hatzinakos and I. Katzela. Tail Probabilities for the Multiplexing of Fractional $\alpha$-Stable Broadband Traffic. To be presented at ICC'01, Helsinki, Finland, 2001.

[8] A. Karasaridis and D. Hatzinakos. Network Heavy Traffic Modeling using $\alpha$-Stable Self-Similar Processes. IEEE Trans. on Commun. (accepted for publication, June 2000).

[9] A. Karasaridis and D. Hatzinakos. On the Modeling of Network Traffic and Fast Simulation of Rare Events using $\alpha$-Stable Self Similar Processes. In Proc. Sign. Proc. Workshop on HOS, Banff, Alberta, 1997.

[10] F.P. Kelly. Models for a Self-Managed Internet. Phil. Trans. R. Soc., A358, pp. 2335-2348, 2000.

[11] T. Konstantopoulos and S.-J. Lin. Macroscopic Models for Long-Range Dependent Network Traffic. Queuing Systems: Theory and Applications, Vol. 28, pp. 215-243, 1998.

[12] W.E. Leland, M.S. Taqqu, W. Willinger and D.V. Wilson. On the Self-similar Nature of Ethernet Traffic (Extended Version). IEEE/ACM Trans. on Networking, Vol. 2, pp. 1-15, 1994.

[13] P. Lévy. Random Functions: General Theory with Special Reference to Laplacian Random Functions. Univ. California Publ. Statist., Vol.1, pp.331-390, 1953.

[14] B.B. Mandelbrot. The Fractal Geometry of Nature. W.H. Freeman, 1982.

[15] B.B. Mandelbrot and J.W. Van Ness. Fractional Brownian Motions, Fractional Noises and Applications. SIAM Rev., Vol. 10, pp. 422-437, 1968.

[16] K.S. Miller and B. Ross. An Introduction to the Fractional Calculus and Fractional Differential Equations. J. Wiley \& Sons, New York, 1993.

[17] C.L. Nikias and M. Shao. Signal Processing with Alpha-Stable Distributions and Applications. Wiley, 1995.

[18] I. Norros. A Storage Model with Self-Similar Input. Queuing Systems, Vol. 16, pp. 387-396, 1994.

[19] K. Park and W. Willinger. Self-Similar Network Traffic and Performance Evaluation. Wiley, 2000.

[20] V. Paxson and S. Floyd. Wide-Area Traffic: The Failure of Poisson Modeling. IEEE/ACM Trans. on Networking, Vol.3, pp. 226-244, 1995.

[21] E. Reich. On the Integrodifferential Equation of Takács (I). Ann. Math. Statist., Vol. 29, pp. 563-570, 1958.

[22] S.G. Samko, A.A. Kilbas, and O.I. Marichev. Fractional Integrals and Derivatives and Ther Applications. (Nauka i Tekhnika, Minsk, 1987), (Translated by Harwood Academic).

[23] G. Samorodnitsky and M.S. Taqqu. Stable Non-Gaussian Random Processes: Stochastic Models with Infinite Variance. London, UK: Chapman and Hall, 1994.

[24] A.V. Skorokhod. Random Processes with Independent Increments. Kluwer, Dordrecht, 1991.

[25] B.J. West and V. Seshadri. Linear Systems with Lev́y Fluctuations. Physica, Vol. 113A, pp. 203-216, 1982.

[26] W. Willinger and V. Paxson. Where Mathematics meets the Internet. Notices of the Amerrican Mathematical Society, Vol.45, pp. 961-970, 1998. 